\title{
Spectrophotometric Studies of Indolic Compounds from Vinca
} Minor L.

\author{
Ana-Maria DUMITRESCU(NECULAI) \\ Phd Student, IOSUD Carol Davila, Bucharest, Romania \\ Gabriela STANCIU \\ "Ovidius" University of Constanta, Department of \\ Chemistry and Chemical Engineering, Constanta, Romania \\ Rodica SIRBU \\ "Ovidius" University of Constanta, Faculty of Pharmacy, \\ Constanta, Romania \\ Florica BUSURICU \\ "Ovidius" University of Constanta, Faculty of Pharmacy, Romania
}

\section{Abstract}

The most medically representative plant in the Apocynaceae family is Vinca minor. Vinca minor is a perennial, herbaceous plant, commonly known as Saschiu. In the present research we aimed to isolate the indole compounds from Vinca minor L. and to carry out some physico-chemical studies on these compounds: UV-VIS spectroscopy and the determination of the polyphenols content using Folin-Ciocâlteu method. The spectrophotometric study of the alcoholic plant extracts obtained from the leaf and stem of Vinca minor was performed using the UV-VIS spectrophotometric method and a VWR UV630PC double beam spectrophotometer. Both samples of plant alcoholic extracts obtained from the leaf and from the stem of Vinca minor L. had the specific absorption maxima detected in the range $225-350 \mathrm{~nm}$ and the absorbance maximum in both cases was 3.5 (u.a- absorbency units). The total content of polyphenols was determined by the Folin-Ciocâlteu method from alcoholic extracts of different concentrations: 40\%, 70\%, 96\% (T40, T70, T96, F40, F70, F96) obtained from the leaf and stem of Vinca minor plant using the spectrophotometer model JASCO- 550 UV VIS. Regarding the alcoholic extracts obtained from the leaf of Vinca minor L., the concentrations of polyphenols were between $812.50 \mathrm{mg} / 100 \mathrm{~g}$ pv and $1737.50 \mathrm{mg} / 100 \mathrm{~g} \mathrm{pv}$ and and in the case of alcoholic extracts obtained from the strain, the results were between 1525.00-3962.50 mg GAE / $100 \mathrm{~g}$ pv, results that were in accordance with the literature.

Keywords: Vinca minor, bioactive substances, UV-VIS spectrophotometric method, Folin-Ciocâlteu method 


\section{Introduction}

In recent decades, new alternative therapies using drugs containing active principles of natural origin, have been a topical field of pharmaceutical research. One of the objectives of these lines of research was to obtain bioactive compounds from natural resources. Plants are the raw material from which various active principles can be extracted, which can be used in the treatment of various diseases. Indole chemistry is a fascinating and complex field. The foul-smelling indole molecule is the cornerstone of a wide variety of natural and chemical products. Moreover, some indole derivatives are vital pharmaceuticals or important intermediates. Indole ring compounds belong to the class of aromatic heterocyclic compounds with a heteroatom and the indole ring is the most widespread heterocycle. Other indole ring alkaloids of particular importance in medicine are Vinca alkaloids [Zimerman H.J., 1999]. Vinca alkaloids inhibit cell division by blocking mitosis, also inhibiting purine and RNA synthesis and ultimately causing the death of rapidly dividing cells. Vincristine and Vinblastine, two important indole alkaloids were isolated from Vinca Rosea L., and their extracts have antitumor activity. Vinorelbine is a semi-synthetic indole alkaloid derived from plant extracts from Vinca minor L. Vincristina was approved for use in cancer chemotherapy in 1963, Vinblastine in 1965, and Vinorelbine in 1994 [Bohannon R.A., 1963]. They have become major components of many combined anticancer regimens, used especially in the treatment of acute leukemia, Hodgkin's disease and other lymphomas, various sarcomas, Wilms tumor, neuroblastoma and breast and lung cancers. Vinca alkaloids are given intravenously, usually at intervals of one or two weeks in cycles with other agents. Vinca alkaloids are available in generic forms and under the trade names Oncovin (vincristine), Velban (vinblastine), Navelbine (vinorelbine) and Oxybral (vincamine) [DeLeve L.D., 2013].

Vincamine, another extremely important indole alkaloid in medicine, was isolated from Vinca minor L., found under the popular name of Saschiu. Vinca minor L. is a perennial, indigenous plant that grows spontaneously, found in our country in the alpine and subalpine area. To date, more than 50 indole alkaloids have been isolated from the aerial parts of this plant, some of which have quaternary structures such as 4-methylraucubaininium chloride, 4-methylstrictamine chloride and 4methylacamycinium chloride. This perennial plant contains indole alkaloids of the monomeric eburnamine type, including vincamine [Vas A., 2005] which has modulatory effects on brain circulation. In folk medicine, vincamine is used internally to treat circulatory disorders, cerebral circulatory deficiencies and support for brain metabolism [La Gow B., 2004]. A large amount of clinical evidence indicates a favorable effect of vincamine in a number of conditions in elderly patients, such as memory disorders, vertigo, transient ischemic deficiencies and headaches. Vincamine increases cerebral blood flow, oxygen consumption, and glucose utilization [Blumenthal M. 1998]. At the skin level, vincamine contributes to the healing of bleeding ulcers, open wounds, bruises, contusions, by speeding up the healing process, [Fischhof P.K.,1996]. 
In the present paper the research hypothesis started from obtaining the indole compounds from Vinca minor L. found in abundance in our country. Starting from the extraction of these compounds of pharmaceutical interest, we analyzed the physicochemical properties of indole compounds from the leaf and stem of plant Vinca minor plant. The general objective of this research is to isolate the indole compounds from the Vinca minor L. and to perform physico-chemical studies on these compounds: UVVIS spectroscopy and determination of the total polyphenol content using the FolinCiocâlteu method. This paper proposes an original approach aimed at achieving the following scientific objectives:

Obtaining and carrying out a biotechnical process of extraction of indole compounds from the leaf and stem of the Vinca minor L. plant.

Characterization of the extraction products of Vinca minor L. by comparative physicochemical studies, using different methods of spectrophotometric analysis (absorption in UV-VIS and determining the polyphenol content using the Folin-Ciocâlteu method.

\section{Material and Methods}

\section{Plant materials}

The plant material under study consists of the leaf and stem of Vinca minor plant, harvested locally, Dobrogea area, depending on their growing periods. The leaves and stems of the plant were selected manually and subjected to a treatment that consisted of repeated washings with drinking and distilled water. The plant material was dried in air, at ambient temperature, in the dark. After drying, the plant material was crushed into powder and stored in paper bags as needed.

\section{Preparation of plant extracts}

The dried and crushed plant material was extracted with ethyl alcohol: 40\%, 70\%, $96 \%$ alcoholic extractive solutions obtained from the leaf and stem of Vinca minor plant were prepared according to the 10th edition of the Roman Pharmacopoeia (F.R. $\mathrm{X}$.). This process involved crushing $10 \mathrm{~g}$ of plant product from the leaf and stem of Vinca minor L. plant, to which was added ethyl alcohol of $40 \%, 70 \%$ and respectively $96 \%$ to $100 \mathrm{~mL}$ (Ratio 1:10). The extracts were left for 10 days in optimal conditions, away from light and moisture, in a cool place. During the 10 days, the extracts were carefully monitored and shaken 2-3 times a day. At the end, they were filtered using cotton cloth filters so that they could be separated from the plant material. The liquid collected on filtration was stored in sterile, dark containers.

\section{Chemicals}

All used reagents were of analytical reagent grade. Gallic acid, Folin - Ciocalteu reagent, metanol p.a. and 20\% anhydrous sodium carbonate were purchased from Sigma-Aldrich, Germany. The solution of gallic acid (standard phenolic compound) $1 \times 10^{-2} \mathrm{~mol} \times \mathrm{L}^{-1}$ was prepared by dissolving $0.1881 \mathrm{~g}$ of gallic acid in $100 \mathrm{~mL}$ ethanol. Folin - Ciocalteu reagent was diluted with distilled water 1:2 (V:V). 


\section{Work equipment}

Usual laboratory utensils and glassware;

Analytical balance;

VWR UV-630PC spectrophotometer;

JASCO-550 UV VIS model spectrophotometer.

\section{Spectrophotometric study of plant extracts obtained from the leaf and stem of the plant Vinca minor $L$.}

The analysis were carried out in the laboratories of the Pharmacy Faculty of Ovidius University of Constanta. The spectrophotometric study of alcoholic plant extracts obtained from the leaf and stem of Vinca minor plant was performed using the UV-VIS spectrophotometric method. A VWR UV-630PC double beam spectrophotometer was used for this analysis. The measurements were performed at room temperature, and the spectral range studied was in the range of 200-400 nm, with specific absorption maxima detected in the range of 225-350 nm. For this analysis, a double-beam VWR UV-630PC spectrophotometer was used using halogen and deuterium sources for visible materials and ultraviolet radiation, at a controlled temperature, $25^{\circ} \mathrm{C}$, using square quartz cuvettes with an optical trajectory of $1 \mathrm{~cm}$. The determination was performed by applying the Bouguer-Lambert-Beer law, known by the linear dependence of the absorbance on the sample concentration. The measurements were performed in triplicate and the error associated with the concentration was the standard deviation obtained.

\section{Study by IR spectroscopy of $70 \%$ alcoholic extracts obtained from the leaf of Vinca minor L. plant}

To identify the chemical structure of indole compounds extracted from the leaf of Vinca minor L., FT-IR spectrophotometric analysis was used. By analogy with the spectra obtained by the UV-VIS method, the IR spectrum is the graphical representation of the percentage of energy absorbed (absorbance or transmittance) depending on the wavelength, in $\mu \mathrm{m}$, or the frequency expressed in $\mathrm{cm}^{-1}$ (wavelength). The spectra were recorded using a FT-IR spectrophotometer model JASCO FT-IR 4200. Standard vincamine ( $0.5 \mathrm{~g})$ was dissolved in $20 \mathrm{~mL}$ of methanol and diluted to $50 \mathrm{~mL}$ with $\mathrm{NaOH} 1 \mathrm{M}$ solution. The final solution was refluxed for 8 hours at $80^{\circ} \mathrm{C}$, after which it was found that pure vincamine (VINC) was partially degraded into degradation products (RV).

\section{Determination of total polyphenol content}

In this study was identified and determined the total phenol content by FolinCiocâlteu method of alcoholic extracts of different concentrations: 40\%, 70\%, 96\% (T40, T70, T96, F40, F70, F96) obtained from the leaf and stem of Vinca minor. 


\section{Folin-Ciocâlteu method}

For the detection of a wide range of compounds with antioxidant action (polyphenols) from a wide variety of plant species, the simplest and most used method is the FolinCiocâlteu method. The basic mechanism of this method is represented by an oxidation / reduction reaction, in which the metal ion is reduced and the phenolic group is oxidized. By using this method the following classes of organic compounds can be identified: flavones, isoflavones, flavanones, flavonols, pro-anthocyanidins, anthocyanins and phenolic acids.

The total phenolic content was determined using the Folin-Ciocâlteu method [14-15]. In a $50 \mathrm{~mL}$ volumetric flask was introduced Vi into $\mathrm{mL}$ of the extracts prepared for

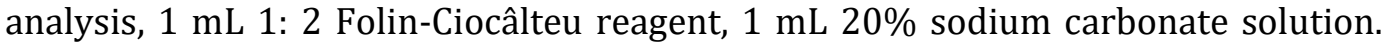
After homogenization, the sample was allowed to stand for 10 minutes, after which the contents of the sample were labeled with distilled water and left for 30 minutes at room temperature to stabilize the color. The absorbance was read using the Jasco 550 UV VIS spectrophotometer at $681 \mathrm{~nm}$. To calculate the concentration of total phenols, expressed in mg of gallic acid (GAE) per $100 \mathrm{~g}$ of dried plant material, equation (1) was applied:

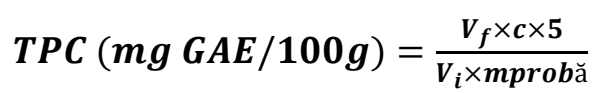

Where, $\mathrm{c}$ represents the concentration ( $\mathrm{mg} / \mathrm{L}$ ) read on the calibration curve, $\mathrm{Vf}$ is the volume of filtered alcoholic extract of each sample, $\mathrm{Vi}$ is the volume of sample added to the $50 \mathrm{~mL}$ rated flask for determination and the sample is the mass of dry plant material (leaf and stem) subjected to maceration.

The samples were prepared in triplicate and the average absorbance value was obtained. The total phenol content was reported as gallic acid equivalents by reference to the linear equation of the standard curve. Then, the total phenolic content was expressed as milligrams of gallic acid equivalent per $100 \mathrm{~g}$ of plant product (mg GAE / $100 \mathrm{~g}$ of plant product).

\section{Results and Discussions}

\section{UV-VIS spectrophotometric study}

The following figures (Fig. 2., Fig. 3., Fig. 4., Fig. 5.) show the chemical structures of indole alkaloids with major importance in the medical and pharmaceutical field. Through the UV-VIS spectrophotometric analysis performed by us, the structure of the indole compounds is confirmed [Neuss N., 1980]. 


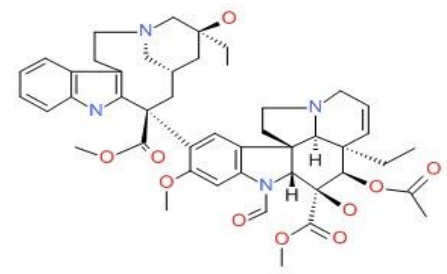

Fig. 2. The chemical structure of Vincristine

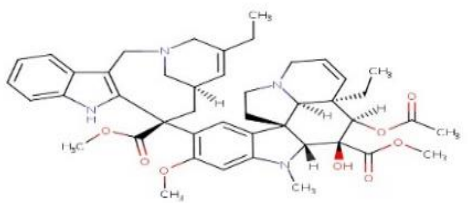

Fig. 4. The chemical structure of Vinorelbine

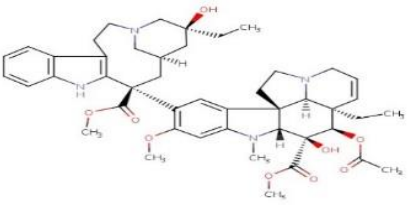

Fig. 3. The chemical structure of Vinblastine

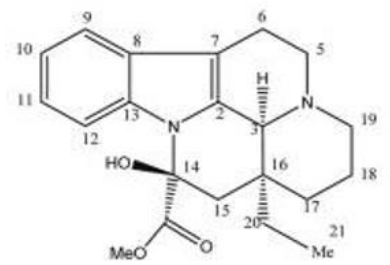

Fig. 5. The chemical structure of Vincamine

In Fig. 6. and Fig. 7. are representated the superimposed UV-VIS absorption spectra obtained for the samples of alcoholic extracts made from the leaf and stem of the Vinca minor plant.

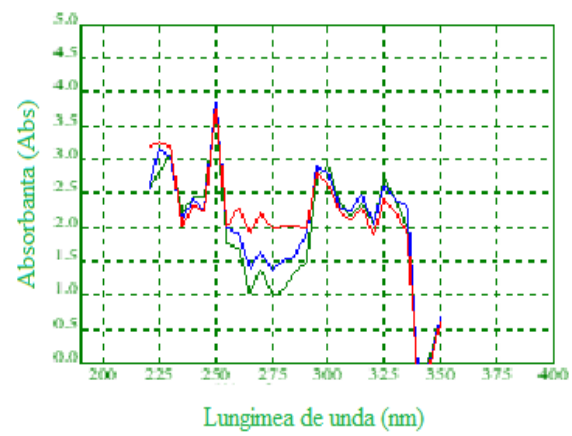

Fig. 6. Overlapping absorption spectra of alcoholic extracts of $40 \%, 70 \%$ and $96 \%$ concentration obtained from the leaf of the Vinca minor plant

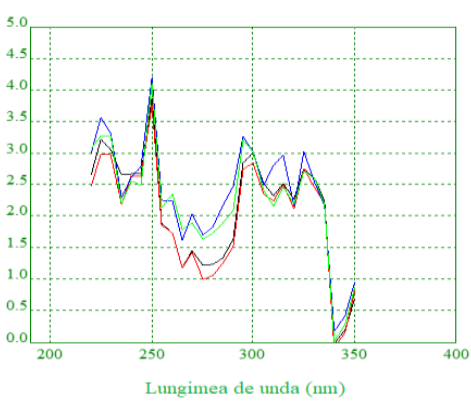

Fig. 7. Overlapping absorption spectra of alcoholic extracts of $40 \%, 70 \%$ and $96 \%$ concentration obtained from the stem of the Vinca minor plant

At the same time, we considered it important to readjust the absorption spectra on different concentration levels for the extracts from the leaf of Vinca minor plant. In 
Figs. 8., 9. and 10. are represented the unique spectra for the samples of alcoholic extracts of concentration 40\% (Fig. 8.), 70\% (Fig. 9.) and 96\% (Fig. 10.) obtained from the leaf of the Vinca minor plant. The spectra obtained in UV-VIS are consistent with the data from the literature [Wootton A., 2011].

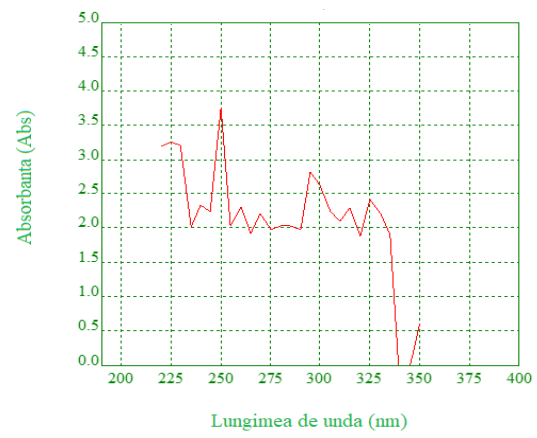

Fig. 8. Absorption spectrum of Fig. 9. Absorption spectrum of alcoholic alcoholic extract with a concentration extract with a concentration of $70 \%$ of the

of $40 \%$ of the leaf of the Vinca minor leaf of the Vinca minor plant plant

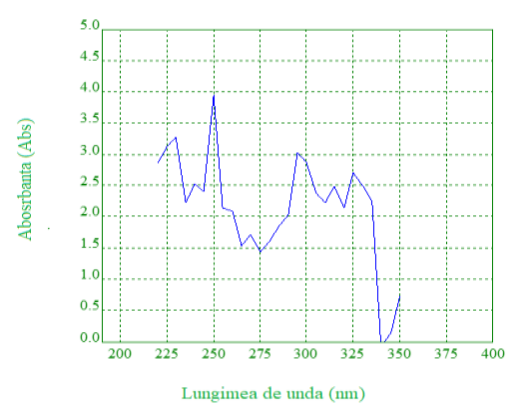

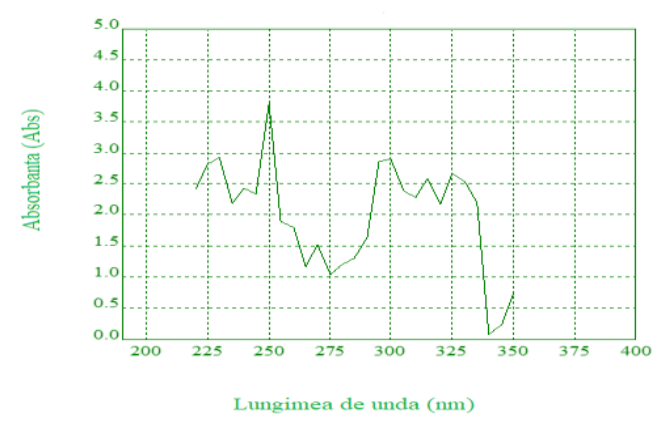

Fig. 10. Absorption spectrum of alcoholic extract with a concentration of $96 \%$ of the leaf of the Vinca minor plant

In the spectrophotometric study (UV-VIS) performed, the extracts obtained from the leaf and stem of the Vinca minor L. plant were analyzed. Absorbance was measured using VWR UV-6300PC spectrophotometer in the range of 200-400 $\mathrm{nm}$. Both in the case of samples of plant alcoholic extracts obtained from the leaf and in the case of those obtained from the stem of Vinca minor L., the specific absorption maxima detected were in the range 225-350 $\mathrm{nm}$ and the absorbance maximum was in both cases 3.5 u.a. (units of absorbance). These results confirm the structure of indole compounds extracted from Vinca minor plant, [Amato A., 1983]. 


\section{IR spectrophotometric study}

The degradation process of pure vincamine (VINC) follows the scheme in Fig.1. These degradation products can be identified by spectrophotometric analysis in IR [S. M. Grujić,. 2015]. In Fig. 10. and 11. are represented the IR spectra for standard vincamine (VINC) and the degradation products of vincamine (RV). According to the structure of vincamine in Figs. 10., it is found that the transmittance registers peaks at wave numbers located in the range $3500 \mathrm{~cm}^{-1}-500 \mathrm{~cm}^{-1}$, thus confirming the types of indole connections existing in Vinca minor plant.

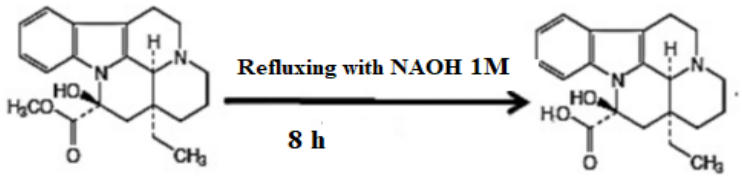

Fig. 1. Degradation scheme of Vincamine in degradation products

The peaks recorded at $3339.24 \mathrm{~cm}^{-1}-319.42 \mathrm{~cm}^{-1}$ indicate the presence of primary amines. The IR spectrum of standard vincamine (VINC) shows a characteristic peak at $1680.38 \mathrm{~cm}^{-1}$, which indicated the presence of the carbonyl group, existing in the structure of vincamine. In the IR spectrum of the degradation products of Fig. 11. appears slightly wider at $3411.88 \mathrm{~cm}^{-1}$, indicating the presence of the hydroxyl group of a carboxylic acid (as a result of hydrolysis produced after degradation). The spectrum obtained is consistent with data from the literature [Ahmed A.B., 2016].

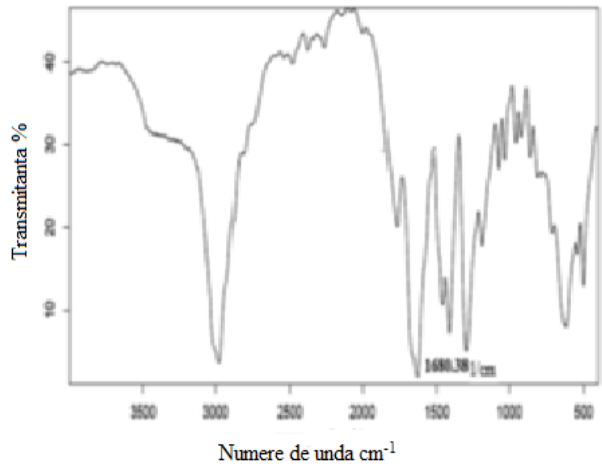

Fig. 10. IR spectrum for standard vincamine (VINC)

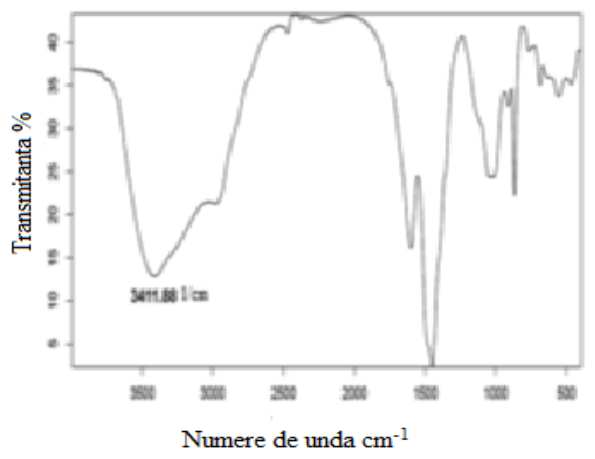

Fig. 11. IR spectrum for vincamine degradation $(\mathrm{RV})$ products

\section{Total phenolic content (TPC)}

Spectral analysis was also used to determine the total polyphenol content in plant extracts of Vinca Minor L, by using the Folin-Ciocâlteu reagent method [9]. Plant 
samples were named as follows: T40, T70 and T96 - alcoholic extracts from Vinca minor L. plant stem. obtained in alcohol concentration 40\%, 70\%, respectively $96 \%$ and F40, F70, F96-alcoholic extracts from the leaf of Vinca minor obtained in alcohol concentration $40 \%, 70 \%$, respectively $96 \%$. Following the analysis, the following values were obtained for the total polyphenol content, for each extract analyzed, by applying the formula from equation (1) These values are represented in Table 1.

The total polyphenol content of alcoholic extracts obtained from the stem and leaf of Vinca Minor L. plant, between $812.50 \mathrm{mg} / 100 \mathrm{~g}$ pv and $3962.50 \mathrm{mg} / 100 \mathrm{~g}$ pv is in accordance with the literature [Nishibe S., 1996]. In the case of alcoholic extracts obtained from the stem of Vinca minor L., the highest concentration of total polyphenols was found in the alcoholic extract 70\%., followed by the alcoholic extract $40 \%$ and the alcoholic extract $96 \%$. Regarding the alcoholic extracts obtained from the leaf of Vinca minor L., the concentrations of polyphenols were between $812.50 \mathrm{mg}$ / 100g pv and $1737.50 \mathrm{mg} / 100 \mathrm{~g} \mathrm{pv}$.

At the same time, it is observed that the extraction with $70 \%$ alcohol was the most efficient, both in the case of the leaf and in the case of the stem, where it results that ethyl alcohol in concentration of $70 \%$ represents the solvent with the highest extraction power of bioactive compounds.

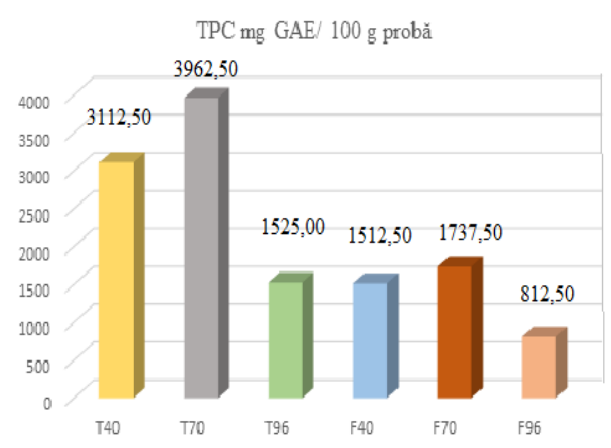

\begin{tabular}{|l|l|}
\hline Sample & $\begin{array}{l}\text { TPC } \\
\text { mg GAE/100 g } \\
\text { pv }\end{array}$ \\
\hline T40 & 3112,50 \\
\hline T70 & 3962,50 \\
\hline T96 & 1525,00 \\
\hline F40 & 1512,50 \\
\hline F70 & 1737,50 \\
\hline F96 & 812,50 \\
\hline
\end{tabular}

Table 1. Concentration of total phenols (TPC) in ethanolic extracts obtained from the leaf and stem of Vinca minor L.

The results of the present study confirm the existence of a higher content of phenolic compounds in the alcoholic extracts obtained from the stem of Vinca minor L., which means that they showed a higher antioxidant activity.

\section{Conclusion}

Indole compounds are a valuable natural source containing numerous physical and chemical properties, associated with the source of the raw material of the analyzed samples but also with the methods of obtaining these compounds. These compounds 
have various fields of applicability: pharmacy, medicine, agriculture. The correct characterization of the physico-chemical properties represents a major role in establishing the field in which indole compounds can be applied. The UV-VIS and IR spectrophotometric studies performed in this research confirms the structure of indole compounds extracted from Vinca minor plant. Similary, the total polyphenol content of the vegetable alcoholic extracts obtained from the leaf and stem of Vinca minor plant was determined using the spectral method Folin-Ciocâlteu. The study carried out on the alcoholic plant extracts of Vinca minor plant, showed a high content of polyphenols both in the case of the stem and in the case of the leaf, a result that confirms their antioxidant action. The total concentrations of polyphenols separated by the Folin-Ciocâlteu method were higher in the case of alcoholic extracts of $70 \%$ concentration obtained both from the stem and from the leaf. We can say that ethyl alcohol in a concentration of $70 \%$ is the solvent with the highest extraction power of bioactive compounds. Following the analysis of the total concentration of polyphenols, it was found that there is a higher content of phenolic compounds in the alcoholic extracts obtained from the stem of Vinca minor plant than in the case of those obtained from the leaf.

\section{References}

[1] Ahmed A.B., Maha M. Abdelrahman, Nada S. Abdelwahab, Fathy M. Salama. Stability-Indicating TLC-Densitometric and HPLC Methods for the Simultaneous Determination of Piracetam and Vincamine in the Presence of Their Degradation Products. Journal of AOAC International, 2016, 99(6) 1490-148

[2] Amato A., Cavazzutti G., Gagliardi L., Profili M., Zagarese V., Chimenti F., Tonelli D., Gattavecchia E., Determination of vincamine by high-performance liquid chromatography with dual-wavelength ultraviolet detection. J. Chromatogr. A, 1983, 270, 387-391

[3] Blumenthal M., The Complete German Commission E Monographs. American Botanical Council in Cooperation with Integrative Medicine Communications. Boston, 1998, 364-365.

[4] Bohannon R.A., Miller D.G., Diamond H.D., Vincristine in the treatment of lymphomas and leukemias. Cancer Res, 1963, 23: 613-621.

[5] DeLeve L.D., Cancer chemotherapy. Kaplowitz N, Deeve L.D, eds. Druginduced liver disease. 3rd ed. Amsterdam: Elsevier, 2013. 549-68.

[6] Fischhof P.K., Moslinger-Gehmayr R., Herrmann W.M., Friedmann A., Russmann D.L. (1996). Therapeutic efficacy of vincamine in ementia. Neuropsychobiology. 1996, 34, 29-35.

[7] La Gow B., PDR for Herbal Medicines, editor. 3rd ed. New Jersey: Thomson $P D R, 2004,632-633$.

[8] Neuss N., The spectrum of biological activities if indole alkaloids. In: Phillipson JD, Zenk MH, editors. Indole and biogenetically related alkaloids. London: Academic Press, 1980, 304-305. 
[9] Nishibe S., Takako T., Takahiko F., Yasukawa K., Takido M., Yasujiro M. and Akira H., Bioactive phenolic compounds from Catharanthus roseus and Vinca minor, Nat. Med, 1996, 378-383.

[10] S. M. Grujić, I. D. Radojević, S. M. Vasić, L. R. Čomić M. D. Topuzović, Antimicrobial and antibiofilm activities of secondary metabolites from Vinca minor L. Applied Biochemistry and Microbiology, 2015, 51, 572-578

[11] Vas A., Gulyas B., Eburnamine derivatives and the brain, Med. Res. Rev., 2005, 25, 737-757.

[12] Wootton A., Ryan L, Food. Res. Int , 2011, 44, (1), 217-224.

[13] Zimerman H.J, The vinca alkaloids. Oncotherapeutic and immunosuppressive agents, Zimmerman HJ., Hepatotoxicity: the adverse effects of drugs and other chemicals on the liver. 2nd ed. Philadelphia: Lippincott, 1999, 692-695. 